\title{
High aerobic intensity training and psychological states in patients with depression or schizophrenia
}

\author{
Jørn Heggelund ${ }^{1,2}$, Kim Daniel Kleppe ${ }^{3}$, Gunnar Morken ${ }^{2,4}$ and Einar Vedul-Kjelsås ${ }^{2,4}$ \\ 1 Division of Psychiatry, Department of Østmarka, St. Olavs University Hospital, Trondheim, Norway \\ 2 Department of Neuroscience, Faculty of Medicine, Norwegian University of Science and Technology, Trondheim, Norway \\ ${ }^{3}$ Hamar District Psychiatric Centre, Innlandet Hospital Trust, Ottestad, Norway \\ ${ }^{4}$ Division of Psychiatry, Department of Research and Development (AFFU), St. Olavs University Hospital, Trondheim, Norway
}

Edited by:

Jan Hoff, Norwegian University of Science and Technology, Norway

\section{Reviewed by:}

Eivind Wang, Norwegian University of Science and Technology, Norway

Øyvind Storen, Telemark University

College, Norway

\section{*Correspondence:}

Jørn Heggelund, Department of Østmarka, Division of Psychiatry, St. Olavs University Hospital, Box 3008

Lade, Trondheim N-7441, Norway e-mail: Jorn.Heggelund@ntnu.no

\begin{abstract}
Aim: To explore changes in psychological states in response to a bout of high aerobic intensity training (HIT) in patients with depression or schizophrenia compared to healthy individuals.
\end{abstract}

Methods: After familiarization training of HIT, 20 patients with schizophrenia, 13 patients with depression, and 20 healthy individuals performed a no-training day followed by a training day. HIT was $4 \times 4$ min intervals at $85-95 \%$ of peak heart rate, intermitted by 3 min active rest periods at $70 \%$ of peak heart rate. Self-evaluation questionnaires of positive affect, negative affect, state anxiety, well-being, distress, and fatigue were completed before training, $15 \mathrm{~min}$ after, and $3 \mathrm{~h}$ after training. The two latter measures were also completed the no-training day.

Results: All three groups improved in positive affect and well-being 15 min after HIT $(p<0.01)$, but only patients with depression had maintained the effect after $3 \mathrm{~h}(p=0.007$, $p=0.012)$. The duration of the improved positive affect was longer in depression $(p=0.002)$ and schizophrenia $(p=0.025)$ than in healthy individuals $\left(F_{2.50}=5.83, p<0.01\right)$. Patients with depression or schizophrenia had reduced distress and state anxiety 15 min after HIT and $3 \mathrm{~h}$ after HIT $(p<0.05)$. The improvement in distress $15 \mathrm{~min}$ after HIT was larger in patients with depression $(p=0.028)$ compared to healthy individuals $\left(F_{2.50}=5.05\right.$, $p<0.01)$. No changes were found during the no-training day $(p>0.05)$.

Conclusion: High aerobic intensity training used as an acute intervention improved positive affect and well-being and reduced distress and state anxiety in patients with depression and schizophrenia.

ClinicalTrials.gov identifier: NCT01310998.

Keywords: exercise, intensity, affect, anxiety, transitory emotions

\section{INTRODUCTION}

Patients with severe mental illnesses suffer from long-term mental health problems that often persist in spite of extensive psychiatric treatments. Thus, acute affective improvements and relief of troubling symptoms may be beneficial, even though they would persist for a limited period of time. Improved self-reported affectivity such as mood enhancements, increased subjective well-being, decreased state anxiety, decreased negative affect, and decreased psychological stress may be brought about by aerobic endurance training in both healthy individuals and patients with severe mental illness (1-3). These affective improvements normally persist for 2-4h following the cessation of exercise (4), but mood enhancements up to $12 \mathrm{~h}$ post-exercise have been reported (5).

To maximize the affective post-exercise improvements, it has been recommended that the exercise intensity is self-selected rather than prescribed $(2,6)$. Furthermore, increasing intensity is associated with reduced positivity of affect (7). These recommendations are somewhat in contrast to recommendations for increasing aerobic fitness in terms of peak oxygen uptake $\left(\mathrm{VO}_{2 \text { peak }}\right)$. High aerobic intensity training (HIT) performed as $4 \times 4$ min intervals at $85-95 \%$ of the maximal heart rate improves $\mathrm{VO}_{2 \text { peak }}$ more than long slow distance training at $70 \%$ of the maximal heart rate $(8-11)$. HIT also improves $\mathrm{VO}_{2 \text { peak }}$ in patients with schizophrenia and could be used as an intervention to reduce the risk of cardiovascular disease in a high risk group, such as patients with severe mental illnesses (12-14). Despite a broad understanding of physiological mediators of improved $\mathrm{VO}_{2 \text { peak }}$ after HIT, it is unknown whether HIT yields supplementary shortterm psychological benefits. Long-term reductions in depressive score are plausible after regular HIT (14). Merely, a few studies have explored interval based exercise as an intervention to improve psychological states (7). Thus, it is reasonable to explore the influence of HIT on post-exercise psychological states.

The acute psychological benefits of aerobic exercise are widely explored in healthy individuals but to a lesser degree in patients with schizophrenia and depression. Patients with depressive 
disorders may score relatively high on negative affect, distress, fatigue, and state anxiety whereas low on positive affect and well-being, contrary to healthy individuals (15). Patients with depression may therefore have a larger window of improvement compared to healthy individuals. Decreased state-anxiety and negative affect along with improved positive well-being have been reported for patients with depression and anxiety $(2,16)$. Conversely, patients with schizophrenia may not be perceptive of psychological states and are known to have a lack of insight into their illness (17). Eight weeks of HIT did not improve chronic symptoms of schizophrenia (12). Acute improvements in state anxiety, psychological stress, and well-being have though been reported (3), and observations suggest that patients are less irritable, depressive, and psychotic as well as more socially interested and competent on the days they have exercised (18). Patients have also reported use of exercise as a coping strategy to deal with positive symptoms (19). HIT may therefore have important therapeutic benefits for patients with depression or schizophrenia. Thus, it is paramount to compare these groups to decide whether patients respond differently from healthy individuals and whether differences in responses exist between patient groups.

To explore the acute psychological benefits of HIT, we included patients with depression, schizophrenia, and healthy individuals to a controlled clinical trial. We hypothesized that a bout of HIT would improve psychological states $15 \mathrm{~min}$ and $3 \mathrm{~h}$ post-exercise, and patients would have greater acute benefits of HIT than healthy individuals.

\section{MATERIALS AND METHODS \\ SUBJECTS}

Fifty-three participants volunteered to take part in one of three study groups: (1) patients with schizophrenia or psychotic disorders, (2) patients with depression, or (3) healthy individuals. Patients were in- and out-patients at the University hospital psychiatric department. The schizophrenia/psychotic group was 20 patients with ICD-10 schizophrenia, schizotypal, or delusional disorders (ICD-10, F20-29) (20). The group of patients with depression comprised 1 with bipolar depression (F31.3), 11 with depressive episode (F32) or recurrent depression (F33), and 1 with dysthymia (F34.1). Twenty healthy controls with no history of psychiatric illness and with a training frequency less than three times per week were enrolled. Characteristics of the subjects are presented in Table 1.

\section{INSTRUMENTS AND PROCEDURES}

During a period of two consecutive days, each participant completed self-report questionnaires' about their psychological states. A bout of HIT was performed on the second day. Questionnaires' were completed $10 \mathrm{~min}$ before (pre-) HIT, $15 \mathrm{~min}$ after (post-) HIT, and $3 \mathrm{~h}$ after HIT. The two latter measures were also completed at the same hour on the first day (no-training day). Participants were instructed to refrain from exercising 2 days before entering the data acquisition.

State anxiety was measured by the State-Trait Anxiety Inventory (STAI) form X-1 (21). A 12-item short form of the original 20 -items was used (22). The STAI is a self-evaluation questionnaire and the instruction is to indicate how the subject feels at a
Table 1 | Characteristics of the subjects

\begin{tabular}{lccc}
\hline & $\begin{array}{c}\text { Schizophrenia } \\
(\boldsymbol{n = 2 0 )}\end{array}$ & $\begin{array}{l}\text { Depression } \\
(\boldsymbol{n = 1 3 )}\end{array}$ & $\begin{array}{c}\text { Healthy } \\
(\boldsymbol{n = 2 0 )}\end{array}$ \\
\hline Men/women, $n$ & $13 / 7$ & $8 / 5$ & $10 / 10$ \\
Age (years), mean (SD) & $37.7(11.8)$ & $41.0(10.9)$ & $40.8(11.8)$ \\
Body weight (kg), mean (SD) & $88.9(17.3)$ & $87.87(26.6)$ & $78.9(14.2)$ \\
BMI (kg m ${ }^{-2}$ ), mean (SD) & $24.79(3.99)$ & $24.95(7.03)$ & $22.42(3.41)$ \\
Inpatient/outpatient, $n$ & $10 / 10$ & $2 / 11$ & \\
ICD-10 diagnoses, $n$ & & & \\
Schizophrenia & 11 & & \\
Schizotypal disorder & 1 & & \\
Delusional disorder & 2 & & \\
Schizoaffective disorder & 5 & & \\
Unspecified non-organic & 1 & & \\
psychosis & & & \\
Bipolar depression & & & \\
Depressive disorder & & & \\
Dysthymia & & & \\
\hline
\end{tabular}

Difference in inpatient/outpatient distribution between patient groups $(p<0.05)$.

particular moment in time. The scale ranges from 1 to 4 . When evaluating the scores, six of the items are reversed so that higher scores indicate higher levels of anxiety. The test-retest correlations range from 0.16 to 0.54 in college students (21). A low level of reliability is expected since the scale reflects the influence of transient situational factors.

Positive affect and negative affect were measured by the Positive and Negative Affect Schedule (PANAS). The PANAS incorporates 10 -items of positive affect and 10 -items of negative affect. The scale ranges from 1 to 5 and higher score indicate higher perception of the factor. The subjects were asked to evaluate whether they experienced a particular emotion in the present moment. The positive affect and negative affect scores correlate negatively and positively, respectively, with the Beck Depression Inventory (BDI) $(15,23)$. Test-retest intraclass correlation is 0.79 and 0.93 for the positive and negative scales, respectively (24).

Positive well-being, psychological distress, and fatigue were measured by the Subjective Exercise Experiences Scale (SEES). SEES is a 12-item questionnaire that is validated to measure effects of exercise on psychological states (25). Each subscale contains the scores from four items and the scale ranges from 1 to 7 . Higher scores indicate a higher perception of the particular factor.

Borg rating of perceived exertion (RPE) was applied to determine subjects' perception of physical exertion during HIT and $\mathrm{VO}_{2 \text { peak }}$ testing (26). The scale ranges from 6 to 20. Six indicate "no exertion at all" whereas 20 is "maximal exertion."

\section{HIGH AEROBIC INTENSITY TRAINING}

High aerobic intensity training was performed on a treadmill according to the procedures described elsewhere $(8,12)$. Four minutes intervals at a fixed workload were repeated 4 times, intermitted with $3 \mathrm{~min}$ active break periods between each interval. The intensity was $85-95 \%$ of peak heart rate $\left(\mathrm{HR}_{\text {peak }}\right)$ during intervals and similar to warming-up work load (i.e., taxing about $70 \%$ of $\mathrm{HR}_{\text {peak }}$ ) during break periods. Subjects either walked or ran a 
minimum of $5 \%$ incline. Warm-up was $5 \mathrm{~min}$. Heart rate was continuously measured using a Polar S610i heart rate monitor (Polar Electro, Finland). Heart rate and RPE was collected 3 min within each interval. All subjects performed a familiarization period of 3 HIT bouts within 2 weeks prior to the study. During familiarization training, the speed and incline were adjusted to attain the exact heart rate intensity (Table 2 ).

All subjects performed a test of $\mathrm{HR}_{\text {peak }}$ in order to calculate the HIT intensity (Table 3). The test was carried out as HIT interval training. The first interval was performed without an experience of leg stiffness. After a $3 \mathrm{~min}$ active rest, the second interval was carried out with $>1 \mathrm{~km} \mathrm{~h}^{-1}$ incensements each minute until exhaustion (preferentially within 3-5 min). $\mathrm{HR}_{\text {peak }}$ was the highest recorded HR. A measure of blood lactate was taken $1 \mathrm{~min}$ after discontinuation using a Lactate Pro blood lactate test meter (Arkray, Inc, Japan). A peak test was accepted at a blood lactate level above $8 \mathrm{mmol} \mathrm{L}^{-1}$. Borgs RPE was collected at discontinuation of the test.

\section{STATISTICAL ANALYSES}

Within-group changes were analyzed using paired $t$-tests. Oneway between-groups ANOVA was used to analyze baseline differences and differences in change between the three groups. Separate analysis were performed on the delta changes (pre- to $15 \mathrm{~min}$ post-HIT) and (pre- to $3 \mathrm{~h}$ post-HIT). LSD or Tamhane's post hoc analyses were performed during significant main analysis. Cohens's $d$ effect sizes were calculated using the formula described in Nakagawa and Cuthill (27). The effect was considered small, medium, and large at $0.2,0.5$, and 0.8 , respectively. Chi-square test was used to evaluate categorical data.

Statistical significance was reached at $p<0.05$. Data are described as mean and SD, unless otherwise noted. IBM SPSS Statistics, version 20 (SPSS Inc.), was applied to analyze results.

The study was approved by the regional committees for medical and health research ethics, middle Norway and conducted according to the Helsinki declaration. Written informed consent was obtained from all the included patients after the procedures were fully explained.

\section{RESULTS}

Characteristics of the patients are presented in Table 1. There were no differences in age, bodyweight, or BMI between the groups $(p>0.05)$. There were no differences between the three groups in $\mathrm{HR}_{\text {peak }}$, speed, or incline during the peak treadmill test $(p>0.05$; Table 3). All three groups performed the HIT at the prescribed intensity and duration. Work load of the intervals are presented in Table 2.

There were between-group differences at baseline in positive affect $\left(F_{2.50}=9.67, p<0.01\right)$, negative affect $\left(F_{2.50}=17.21\right.$, $p<0.01)$, positive well-being $\left(F_{2.50}=19.84, p<0.01\right)$, psychological distress $\left(F_{2.50}=12.64, p<0.01\right)$, fatigue $\left(F_{2.50}=8.36\right.$, $p<0.01)$, and state anxiety $\left(F_{2.50}=21.74, p<0.01\right)$. Post hoc analyses revealed that the positive affect $(p=0.046$ and $p<0.001)$, negative affect $(p=0.005$ and $p<0.001)$, psychological distress $(p=0.006$ and $p<0.001)$, fatigue $(p=0.012$ and $p<0.001)$, and state anxiety $(p=0.001$ and $p<0.001)$ were different between health individuals and patients with schizophrenia and depression,
Table 2 | Mean (SD) work load of intervals during the high aerobic intensity training.

\begin{tabular}{lccc}
\hline & $\begin{array}{l}\text { Schizophrenia } \\
(\boldsymbol{n}=\mathbf{2 0})\end{array}$ & $\begin{array}{l}\text { Depression } \\
(\boldsymbol{n}=\mathbf{1 3})\end{array}$ & $\begin{array}{l}\text { Healthy } \\
(\boldsymbol{n}=\mathbf{2 0})\end{array}$ \\
\hline Speed $\left(\mathrm{km} \mathrm{h}^{-1}\right)$ & $7.4(1.7)$ & $7.2(1.9)$ & $8.5(2.3)$ \\
Incline $(\%)$ & $5.5(1.3)$ & $6.7(3.4)$ & $6.3(1.0)$ \\
Watt & $95(29)$ & $106(45)$ & $121(29)$ \\
\hline
\end{tabular}

No differences between groups.

Table 3 | Mean (SD) values of the peak heart rate test.

\begin{tabular}{lccc}
\hline & $\begin{array}{l}\text { Schizophrenia } \\
(\boldsymbol{n}=\mathbf{2 0})\end{array}$ & $\begin{array}{l}\text { Depression } \\
(\boldsymbol{n = 1 3 )}\end{array}$ & $\begin{array}{l}\text { Healthy } \\
(\boldsymbol{n}=\mathbf{2 0})\end{array}$ \\
\hline HR $_{\text {peak }}$ (beats $\left.\mathrm{min}^{-1}\right)$ & $177(20)$ & $186(9)$ & $186(12)$ \\
Peak speed $\left(\mathrm{km} \mathrm{h}^{-1}\right)$ & $9.4(2.8)$ & $9.5(2.5)$ & $11.4(3.5)$ \\
Peak incline (\%) & $9.2(3.8)$ & $8.9(3.8)$ & $9.6(3.8)$ \\
Watt & $192(75)$ & $190(86)$ & $218(79)$ \\
La $\left.^{-}(\mathrm{mmol})^{-1}\right)$ & $11.02(3.60)$ & $11.2(2.2)$ & $11.78(3.16)$ \\
BORG RPE & $19(2)$ & $19(1)$ & $19(1)$ \\
\hline
\end{tabular}

No differences between groups.

$H R_{\text {peak }}$, peak heart rate; min, minute; RPE, rating of perceived exertion.

respectively. Positive well-being was different between the healthy individuals and patients with depression $(p<0.001)$ but not between healthy individuals and patients with schizophrenia $(p>0.05)$. The patients with schizophrenia and patients with depression scored differently in positive affect $(p=0.013)$ and positive well-being $(p<0.001)$ but not in negative affect, psychological distress, fatigue, and state anxiety $(p>0.05)$ at baseline.

\section{POSITIVE AFFECT AND POSITIVE WELL-BEING}

All three groups increased their experience of positive affect and well-being from pre- to 15 min post-HIT (Figure 1). The Cohen's $d$ effect size indicated a medium (0.66), large (0.91), and small (0.45) effect on the positive affect in the patients with schizophrenia, patients with depression, and healthy individuals, respectively. The corresponding Cohen's $d$ effect sizes for positive well-being was medium (0.74), large (1.05), and medium (0.58), respectively. After $3 \mathrm{~h}$, the Cohen's $d$ effect sizes for positive affect and positive well-being were still large (0.96 and 0.90$)$ in the patients with depression (Figure 2). The change in positive affect from pre-HIT to $3 \mathrm{~h}$ post-HIT was different between the groups $\left(F_{2.50}=5.83\right.$, $p<0.01$ ), and the post hoc analyses revealed that the change in healthy individuals was different from patients with schizophrenia $(p=0.025)$ and patients with depression $(p=0.008)$.

\section{PSYCHOLOGICAL DISTRESS}

The patients with schizophrenia and depression reduced their experience of psychological distress with a small (0.39) and medium (0.71) Cohen's $d$ effect size, respectively, from pre- to $15 \mathrm{~min}$ post-HIT (Figure 1). The Cohen's $d$ effect size for distress indicated a medium effect $3 \mathrm{~h}$ post-HIT (Figure 2 ) in the patients with schizophrenia (0.48) and depression (0.56). Cohen's $d$ effect 


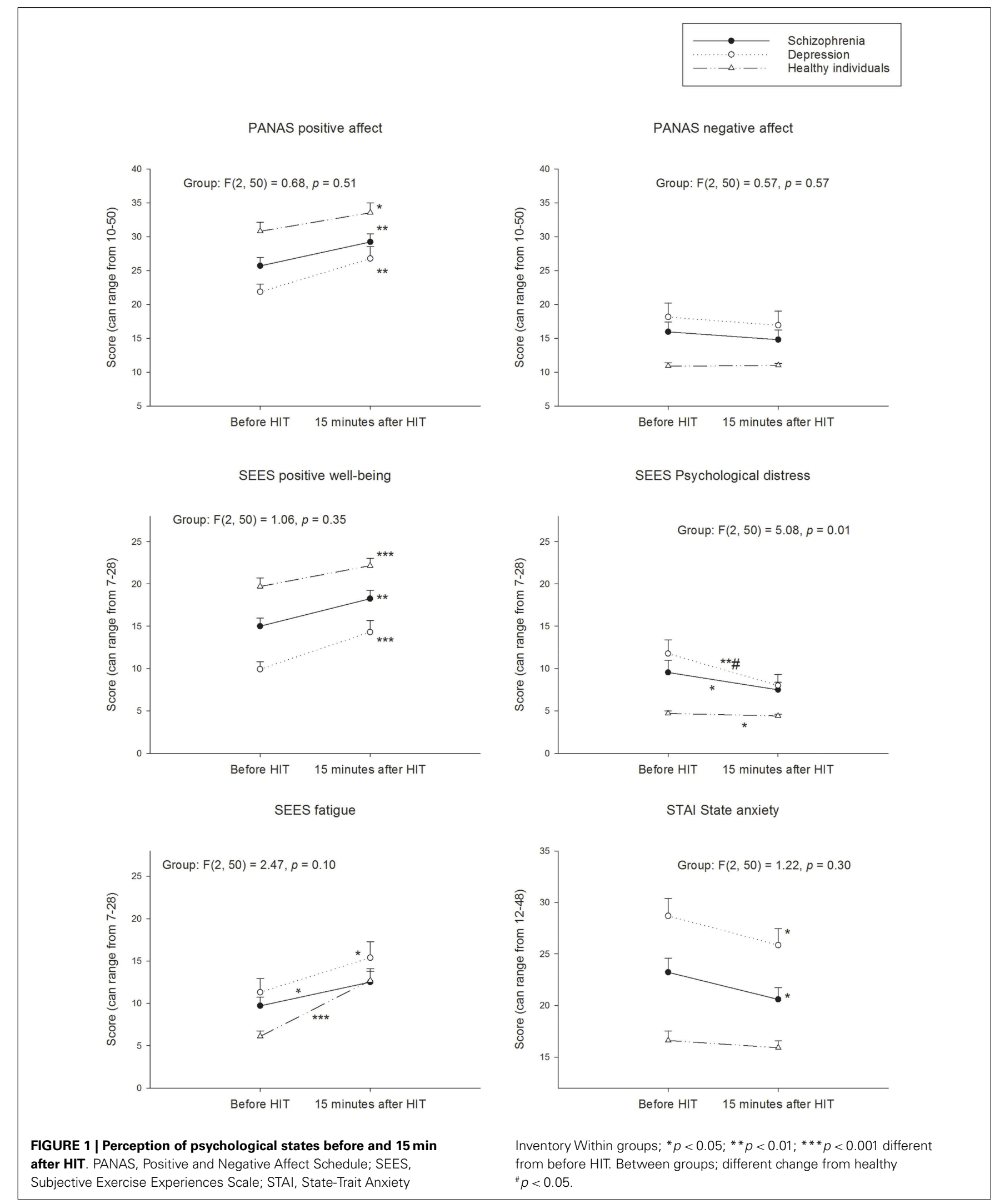


PANAS positive affect

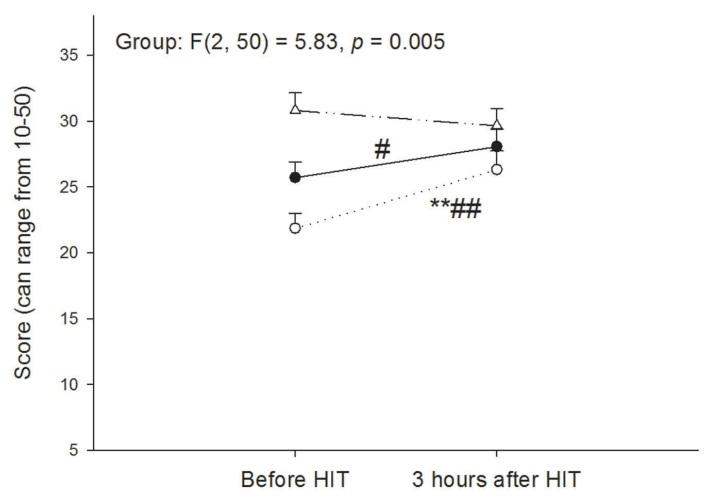

SEES positive well-being

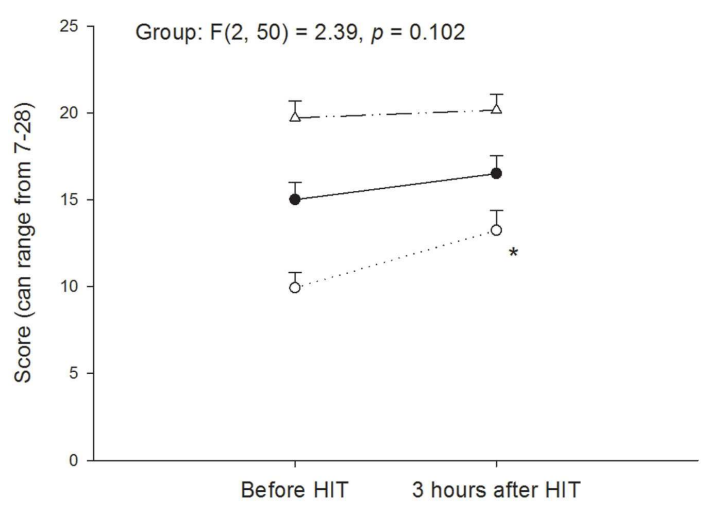

SEES fatigue

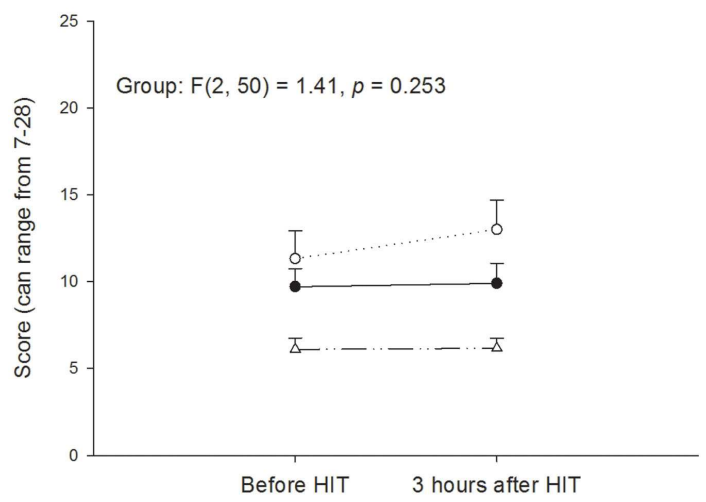

FIGURE 2 | Perception of psychological states before and after $\mathbf{3} \mathbf{h}$

after HIT. PANAS, Positive and Negative Affect Schedule; SEES,

Subjective Exercise Experiences Scale; STAI, State-Trait Anxiety

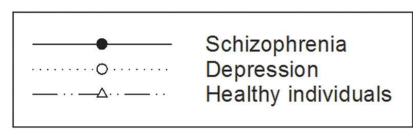

PANAS negative affect

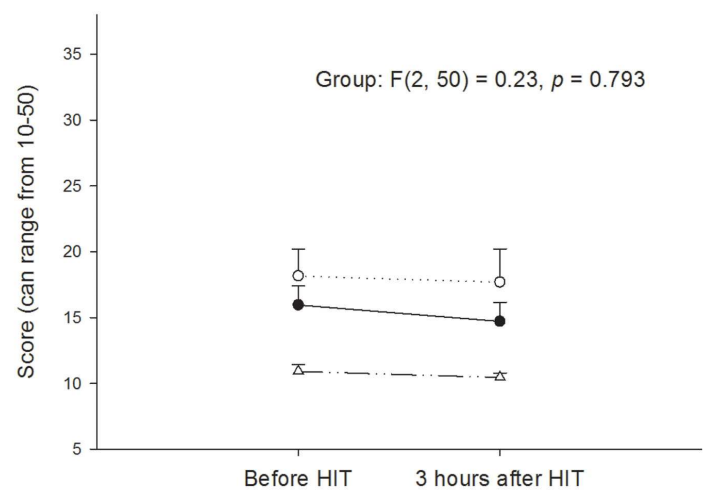

SEES psychological distress

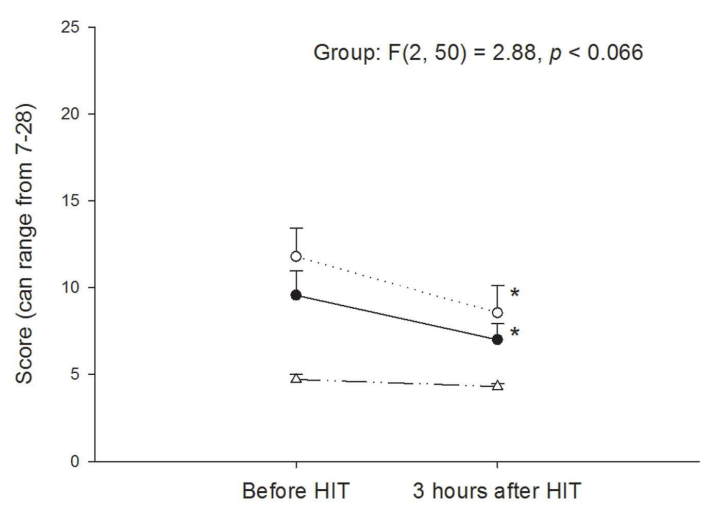

STAI State anxiety

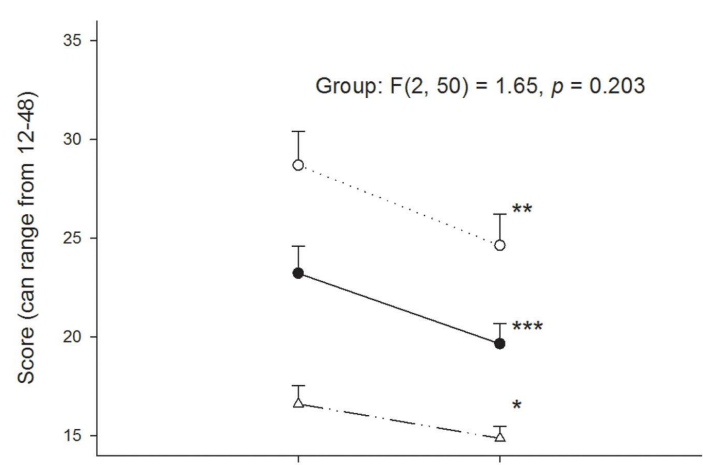

Before HIT 3 hours after HIT

Inventory. Within groups; ${ }^{*} p<0.05$; ${ }^{*} p<0.01$; ${ }^{*}{ }^{*} p<0.001$ different from before HIT. Between groups; different change from healthy $\# p<0.05 ; \# p<0.01$. 
size indicated a small effect (0.26) on distress in the healthy individuals, $15 \mathrm{~min}$ post-HIT. The three groups changed differently in distress from pre-HIT to $15 \mathrm{~min}$ post-HIT $\left(F_{2.50}=5.05\right.$, $p<0.01)$ and the post hoc analyses reviled a difference between the healthy individuals and the patients with depression $(p=0.028)$.

\section{STATE ANXIETY}

The Cohen's $d$ effect sizes for state anxiety for patients with schizophrenia and patients with depression was medium (0.47 and 0.48) $15 \mathrm{~min}$ following the HIT (Figure 1). Cohen's $d$ indicated a medium (0.66) effect in patients with schizophrenia as well as in patients with depression (0.68). The healthy individuals had a higher level of state anxiety immediately before the HIT compared with the no-training day ( $p=0.025$; Cohen's $d=0.59)$. This increase in state anxiety was unchanged from pre- to $15 \mathrm{~min}$ postHIT $(p=0.408)$ but had returned to no-training day levels $3 \mathrm{~h}$ post-HIT as indicated by a reduction in state anxiety from pre-HIT values ( $p=0.038$; Cohen's $d=0.50$; Figure 2$)$.

\section{NEGATIVE AFFECT}

The negative affect was unchanged following HIT in all groups (Figures 1 and 2). The negative affect was already reduced in the patients with depression before the HIT bout compared to the no-training day $(p=0.024$; Cohen's $d=0.59)$.

\section{NO-TRAINING DAY}

During the no-training day, the two measures were not different in either group $(p>0.05)$, which indicate a stable experience of all six psychological state variables.

Patients with schizophrenia $(p=0.038$; Cohen's $d=0.45)$, depression $(p=0.009$; Cohen's $d=0.81)$, and healthy individuals ( $p=0.007$; Cohen's $d=0.48$ ) experienced a lower level of fatigue before performing the HIT session compared to the no-training day.

\section{DISCUSSION}

The results from the present study support the hypothesis that supervised HIT improves the psychological state by increasing positive affect and well-being and reduce state anxiety and distress $15 \mathrm{~min}$ post-exercise in patients with schizophrenia and in patients with depression. The exact mechanism that led to the improved psychological state is not possible to elucidate from this study. Social, cognitive, and biological mechanisms of performing HIT in a supervised fashion in an Exercise Training Clinic contribute to the psychological response. However, when HIT is presented in this clinical wrapping, the high aerobic intensity falls within the range of intensities that improves psychological states beyond baseline levels already $15 \mathrm{~min}$ after cessation. The present study also finds that a prescribed intensity enhances the affective responses. Thus, it is possible to achieve these beneficial affective responses from prescribing an aerobic endurance training program that is developed for optimizing $\mathrm{VO}_{2 \text { peak }}$. Exercising at a high aerobic intensity (i.e., $85-95 \% \mathrm{HR}_{\text {peak }}$ ) is fatigable, as should be expected. All subjects rated the intervals in the range from somewhat hard - very hard (heavy), which is reasonably and at a fair distance from maximal exertion. The perception of fatigue, from the SEES questionnaire, is evident $15 \mathrm{~min}$ after cessation but is attenuated sometime within $3 \mathrm{~h}$.
Patients with depression or schizophrenia sustained the reduction in distress and state anxiety more than $3 \mathrm{~h}$ after HIT but the improved positive affect and well-being did only persist in the patients with depression. The negative affect were unaltered in all groups at both of the post-HIT assessments. Together, these findings replicate previous results suggesting that the psychological benefits are sustained for some hours after the exercise is terminated $(4,5)$. The findings are tentative evidence that patients with depression might have particularly beneficial and sustainable responses in positive affect and well-being after HIT compared to the patients with schizophrenia and the healthy individuals. Patients with depression also have the lowest baseline scores in positive affect and well-being, which provides a large window of improvement. Positive affect and BDI score are in fact negatively correlated, which indicate that patients with enhancements in positive affect also would experience a reduction in BDI scores (23). Further, positive affect is more strongly related to depression than to anxiety (15). Thus, patients with high levels of depression might have important therapeutic benefits from HIT (16). Chamove (18) found a reduction in depression $2 \mathrm{~h}$ after exercise in patients with schizophrenia. Chamove (18) found that patients were "less tense" and "less irritable," which probably reflect similar changes as the reduced distress and state anxiety in the present study. Chamove (18) applied a wider range of physical activities, illustrating that affective improvements likely appear through a multifactorial mechanism.

The between groups analyses confirm some beneficial responses in patients compared to healthy individuals. The duration of the improved positive affect was longer for those with depression or schizophrenia than in the healthy individuals. Further, the $15 \mathrm{~min}$ post-HIT effect on distress was larger in patients with depression compared to healthy individuals. These findings support the hypothesis that the benefits are largest in those with the most unbalanced affect and underscore the particular role of exercise in the treatment of patients with depression (28). It has been suggested that HIT does not improve chronic symptoms in patients with schizophrenia (12). Nevertheless, it now appears that patients are perceptive of certain affective improvements that exceed those of the healthy individuals. This might have important implications for daily functioning and quality of life, particularly at the days of exercise.

The psychological states were stable during the no-exercise day, which supports the influence of HIT. Further, the finding that some variables sustained elevated/reduced $3 \mathrm{~h}$ post-HIT also supports the hypothesis that they were influenced by HIT rather than being spontaneous changes. Studies also suggest that fitness level might influence the psychological response to exercise (7). $\mathrm{VO}_{2 \text { peak }}$ measurements were not collected to explore exact differences between the three groups. However, the speed, incline, and watt load during the peak exercise test and the HIT intervals did not reveal any significant changes between the groups. We also assume that the familiarization training limited some of the affective responses of attending to an exercise regimen for the first time.

This study investigated supervised HIT at the hospitals Exercise Training Clinic. Thus, a combination of social, cognitive, and biological mechanisms likely has been responsible for the improvements (29). Performing supervised HIT exposes the patient to 
a range of psychosocial stimuli such as diversion from stressful stimuli, attention form the coach, improved self-image, feelings of control, social interaction, and social support (29). It is also suggested that exercise improves depression through a neurobiological mechanism. Regular exercise may exert a regulatory influence on the neurotransmitter system or/and affect the hypothalamic-pituitary-adrenal cortical (HPA) axis (29).

This study did not aim to compare HIT with other types of exercise interventions. Thus, it is not possible to advocate the use of HIT merely based on its effect on psychological states. Although the finding that a single HIT session is beneficial, it is appropriate to implement HIT on a regular basis in order to improve fitness and overall health. Better $\mathrm{VO}_{2 \text { peak }}$ is associated with decreased depressive symptoms, quality of life, better capacity to cope with stress as well as reduced mortality from cardiovascular disease (30-33). It is evidential that HIT performed as $4 \times 4 \mathrm{~min}$ intervals induces larger improvements in $\mathrm{VO}_{2 \text { peak }}$ compared to other aerobic endurance training interventions (8-11). This large improvement caused by HIT contribute risk reduction of cardiovascular disease $(12,34)$ and likely influence depressive score (14). Thus, HIT should be implemented in clinical practice to improve $\mathrm{VO}_{2 \text { peak }}$ in patients with severe mental illnesses.

\section{CONCLUSION}

Supervised HIT performed at the Hospitals Exercise Training Clinic increased positive affect and well-being and reduced state anxiety and distress. It is therefore possible that patients could have twofold benefits from HIT. Training effectively to improve $\mathrm{VO}_{2 \text { peak }}$ gave acute psychological benefits. Reductions in distress and state anxiety were sustained for more than $3 \mathrm{~h}$ after HIT and patients with depression also sustained the improved positive affect and well-being. The duration of the improved positive affect was longer for those with depressive and schizophrenia disorders than in the healthy individuals. The reduction in distress was also larger in patients with depression compared to healthy individuals with a low distress at baseline. It is therefore reasonable to conclude that patients with reduced affectivity had benefits from HIT that exceeded those in the healthy individuals.

\section{AUTHOR CONTRIBUTIONS}

Jørn Heggelund, Kim Daniel Kleppe, Gunnar Morken, and Einar Vedul-Kjelsås took part in the concept and design of the study. Jørn Heggelund conducted data analyses and prepared the manuscript. Kim Daniel Kleppe recruited participants and collected data, performed preliminary analyses and preparation of the manuscript. Gunnar Morken assisted data analyses, interpretation, and preparation of the manuscript. Einar Vedul-Kjelsås assisted with data collection, data analyses, and interpretation, and was involved in the preparation of the manuscript. All authors approved the final manuscript.

\section{ACKNOWLEDGMENTS}

We acknowledge the participants who took part in the study. We thank physiotherapist Aksel Skjelbred and psychiatric nurse Kari Kristiansen Herre for the contribution in data acquisition.

\section{REFERENCES}

1. Hale BS, Koch KR, Raglin JS. State anxiety responses to 60 minutes of cross training. Br J Sports Med (2002) 36(2):105-7. doi:10.1136/bjsm.36.2.105

2. Knapen J, Sommerijns E, Vancampfort D, Sienaert P, Pieters G, Haake P, et al. State anxiety and subjective well-being responses to acute bouts of aerobic exercise in patients with depressive and anxiety disorders. Br J Sports Med (2009) 43(10):756-9. doi:10.1136/bjsm.2008.052654

3. Vancampfort D, De Hert M, Knapen J, Wampers M, Demunter H, Deckx S, et al. State anxiety, psychological stress and positive well-being responses to yoga and aerobic exercise in people with schizophrenia: a pilot study. Disabil Rehabil (2011) 33(8):684-9. doi:10.3109/09638288.2010.509458

4. Raglin JS, Wilson GS. Exercise and its effects on mental health. 2 ed. In: Bouchard C, Blair S, Haskell WL, editors. Physical Activity and Health. (Vol. 331-342), Champaign, IL: Human Kinetics (2012). p. 247-57.

5. Sibold JS, Berg KM. Mood enhancement persists for up to 12 hours following aerobic exercise: a pilot study. Percept Mot Skills (2010) 111(2):333-42. doi:10.2466/02.06.13.15.PMS.111.5.333-342

6. Szabo A. Acute psychological benefits of exercise performed at self selected workloads: implications for theory and practice. J Sports Sci Med (2003) 2(3): 77-87.

7. Ekkekakis P, Petruzzello SJ. Acute aerobic exercise and affect: current status, problems and prospects regarding dose-response. Sports Med (1999) 28(5):337-74. doi:10.2165/00007256-199928050-00005

8. Helgerud J, Hoydal K, Wang E, Karlsen T, Berg P, Bjerkaas M, et al. Aerobic high-intensity intervals improve VO2max more than moderate training. Med Sci Sports Exerc (2007) 39(4):665-71. doi:10.1249/mss.0b013e3180304570

9. Rognmo O, Hetland E, Helgerud J, Hoff J, Slordahl SA. High intensity aerobic interval exercise is superior to moderate intensity exercise for increasing aerobic capacity in patients with coronary artery disease. Eur J Cardiovasc Prev Rehabil (2004) 11(3):216-22. doi:10.1097/01.hjr.0000131677.96762.0c

10. Wisloff U, Stoylen A, Loennechen JP, Bruvold M, Rognmo O, Haram PM, et al. Superior cardiovascular effect of aerobic interval training versus moderate continuous training in heart failure patients: a randomized study. Circulation (2007) 115(24):3086-94. doi:10.1161/CIRCULATIONAHA.106.675041

11. Tjonna AE, Lee SJ, Rognmo O, Stolen TO, Bye A, Haram PM, et al. Aerobic interval training versus continuous moderate exercise as a treatment for the metabolic syndrome: a pilot study. Circulation (2008) 118(4):346-54. doi:10.1161/CIRCULATIONAHA.108.772822

12. Heggelund J, Nilsberg GE, Hoff J, Morken G, Helgerud J. Effects of high aerobic intensity training in patients with schizophrenia-A controlled trial. Nord J Psychiatry (2011) 65(4):269-75. doi:10.3109/08039488.2011.560278

13. Hennekens $\mathrm{CH}$. Increasing global burden of cardiovascular disease in general populations and patients with schizophrenia. J Clin Psychiatry (2007) 68(Suppl 4):4-7. doi:10.4088/JCP.0507e12

14. Flemmen G, Unhjem R, Wang E. High-intensity interval training in patients with substance use disorder. Biomed Res Int (2014) 2014:616935. doi:10.1155/ 2014/616935

15. Crawford JR, Henry JD. The positive and negative affect schedule (PANAS): construct validity, measurement properties and normative data in a large non-clinical sample. Br J Clin Psychol (2004) 43(Pt 3):245-65. doi:10.1348/ 0144665031752934

16. Bartholomew JB, Morrison D, Ciccolo JT. Effects of acute exercise on mood and well-being in patients with major depressive disorder. Med Sci Sports Exerc (2005) 37(12):2032-7. doi:10.1249/01.mss.0000178101.78322.dd

17. Linaker OM, Moe A. The COOP/WONCA charts in an acute psychiatric ward. Validity and reliability of patients' self-report of functioning. Nord J Psychiatry (2005) 59(2):121-6. doi:10.1080/08039480510022918

18. Chamove AS. Positive short-term effects of activity on behaviour in chronic schizophrenic patients. Br J Clin Psychol (1986) 25(Pt 2):125-33. doi:10.1111/j. 2044-8260.1986.tb00681.x

19. Faulkner G, Sparkes A. Exercise as therapy for schizophrenia: an ethnographic study. J Sport Exerc Psychol (1999) 21(1):52-70.

20. World Health Organization. The ICD-10 Classification of Mental and Behavioural Disorders: Diagnostic Criteria for Research. (Vol. XIII). Geneva: World Health Organization (1993). 248 p.

21. Spielberger CD, Gorsuch RL, Lushene R, Vagg PR, Jacobs GA. Manual for the State-Trait Anxiety Inventory. Palo Alto, CA: Consulting Psychologists Press (1983). 
22. Bodin T, Martinsen EW. Mood and self-efficacy during acute exercise in clinical depression. A randomized, controlled study. J Sport Exerc Psychol (2004) 26:623-33.

23. Watson D, Clark LA, Tellegen A. Development and validation of brief measures of positive and negative affect: the PANAS scales. J Pers Soc Psychol (1988) 54(6):1063-70. doi:10.1037/0022-3514.54.6.1063

24. Ostir GV, Smith PM, Smith D, Ottenbacher KJ. Reliability of the positive and negative affect schedule (PANAS) in medical rehabilitation. Clin Rehabil (2005) 19(7):767-9. doi:10.1191/0269215505cr894oa

25. McAuley E, Courneya KS. Sport psychology the subjective exercise experiences scale (SEES): development and preliminary validation. J Sport Exerc Psychol (1994) 16(2):163-77. doi:10.1016/j.jbmt.2008.11.005

26. Borg G. Perceived exertion as an indicator of somatic stress. Scand J Rehabil Med (1970) 2(2):92-8.

27. Nakagawa S, Cuthill IC. Effect size, confidence interval and statistical significance: a practical guide for biologists. Biol Rev Camb Philos Soc (2007) 82(4):591-605. doi:10.1111/j.1469-185X.2007.00027.x

28. Rimer J, Dwan K, Lawlor DA, Greig CA, McMurdo M, Morley W, et al. Exercise for depression. Cochrane Database Syst Rev (2012) 7:CD004366. doi:10.1002/14651858.CD004366

29. Buckworth J, Dishman RK, O'Connor PJ, Tomporowski PD. Depression. In: Schrag M, Zavala M, Cox K, Feeney J, Fortney P, editors. Exercise Psychology. Champaign, IL: Human Kinetics (2013). p. 185-219.

30. Gerber M, Lindwall M, Lindegard A, Borjesson M, Jonsdottir IH. Cardiorespiratory fitness protects against stress-related symptoms of burnout and depression. Patient Educ Couns (2013) 93(1):146-52. doi:10.1016/j.pec.2013.03.021

31. Rieck T, Jackson A, Martin SB, Petrie T, Greenleaf C. Health-related fitness, body mass index, and risk of depression among adolescents. Med Sci Sports Exerc (2013) 45(6):1083-8. doi:10.1249/MSS.0b013e3182831db1
32. Sloan RA, Sawada SS, Martin CK, Church T, Blair SN. Associations between cardiorespiratory fitness and health-related quality of life. Health Qual Life Outcomes (2009) 7:47. doi:10.1186/1477-7525-7-47

33. Sui X, Laditka JN, Church TS, Hardin JW, Chase N, Davis K, et al. Prospective study of cardiorespiratory fitness and depressive symptoms in women and men. J Psychiatr Res (2009) 43(5):546-52. doi:10.1016/j.jpsychires. 2008.08.002

34. Heggelund J, Hoff J, Helgerud J, Nilsberg GE, Morken G. Reduced peak oxygen uptake and implications for cardiovascular health and quality of life in patients with schizophrenia. BMC Psychiatry (2011) 11(1):188. doi:10.1186/1471-244X11- 188

Conflict of Interest Statement: The authors declare that the research was conducted in the absence of any commercial or financial relationships that could be construed as a potential conflict of interest.

Received: 16 May 2014; accepted: 11 October 2014; published online: 30 October 2014. Citation: Heggelund J, Kleppe KD, Morken Gand Vedul-Kjelsås E (2014) High aerobic intensity training and psychological states in patients with depression or schizophrenia. Front. Psychiatry 5:148. doi: 10.3389/fpsyt.2014.00148

This article was submitted to Schizophrenia, a section of the journal Frontiers in Psychiatry.

Copyright (C) 2014 Heggelund, Kleppe, Morken and Vedul-Kjelsås. This is an openaccess article distributed under the terms of the Creative Commons Attribution License (CC BY). The use, distribution or reproduction in other forums is permitted, provided the original author(s) or licensor are credited and that the original publication in this journal is cited, in accordance with accepted academic practice. No use, distribution or reproduction is permitted which does not comply with these terms. 